\title{
28 Research Suare \\ Exosomal miR-1246 is Involved in Tumorigenesis by Targeting THRB in Breast Cancer
}

Jie Zhu

Taizhou Central Hospital(Taizhou University Hospital)

\section{Xiaoying Chen}

Hebei North University

Fengxiang $\mathbf{X i}$

Taizhou University

Lei Zhao

Taizhou Central Hospital(Taizhou University Hospital)

\section{Yichao Wang}

Taizhou Central Hospital(Taizhou University Hospital)

Jiaogui Xie ( $D$ 847104404@qq.com )

Taizhou customs

Research

Keywords: breast cancer, miRNA, exosomes, miR-1246

Posted Date: January 9th, 2021

DOl: https://doi.org/10.21203/rs.3.rs-141174/v1

License: (c) (i) This work is licensed under a Creative Commons Attribution 4.0 International License.

Read Full License 


\section{Abstract}

Background: Exosomal miRNAs have drawn increasing attention as tumor biomarkers involving in tumorigenesis due to their stability. The aim of this study was to analyze the role of exosomal miR-1246 in breast cancer.

Methods: The differentially expressed (DE) miRNAs in exosomes from the serum of breast cancer patients and healthy controls were investigated using RNA-seq. The potential pathogenic target genes and functional enrichment of these miRNAs were explored using bioinformatics. Additionally, the role of miR-1246 in migration, invasion and proliferation were investigated in breast cancer cells. The expression of THRB were detected by QRT-PCR. Kaplan-Meier plotter was used to perform survival analysis.

Results: The results showed that the level of exosomal miR-1246 was significantly higher in breast cancer than in the control. MiR-1246 mimic treatment promoted invasion and migration in MDA-MB-231 cells by targeting THRB. Kaplan-Meier survival curves showed that patients with high miR-1246 expression exhibited poor OS compared with patients with low miR-1246 expression, especially those with metastatic breast cancer.

Conclusion: Our study provides a better understanding of exosomal miR-1246 in the process of breast cancer. Exosomal miR-1246 could be a potential prognostic biomarker for breast carcinoma.

\section{Introduction}

Breast cancer is the most widespread kind of cancer in women[1]. Although the prognosis of breast cancer patients has improved considerably, almost one-third of women still die from this disease due to metastasis[2]. With the development of clinical medicine, early diagnosis of breast cancer is very important to improve the therapeutic effect and survival rate. Currently, mammography is a widely used method for breast cancer screening[3], but it is limited by the tumor size and the low radiation utilized on certain patients. Molecular biomarkers, such as the estrogen receptor (ER), progesterone receptor, and human epidermal growth factor receptor (Her2/ErBb2), are used for diagnosis or for predicting outcomes[4,5]. However, these biomarkers are not sufficiently effective or dependable to screen tumor cells isolated from the serum of breast cancer patients. Thus, it is imperative to find more precise serum molecular biomarkers for breast cancer diagnosis.

In recent years, exosomes have been investigated as potential biomarkers in several diseases and health conditions[6-8]. Malignant cells secrete a large number of exosomes, which contain various molecular components, such as microRNAs (miRNAs), proteins, and lipids[9]. MiRNAs are small noncoding RNAs that play regulatory roles in cell proliferation, cell cycle regulation, drug resistance, and other biological processes[10]. Exosomes are nanocarriers that can transfer different cargos, such as miRNAs, to recipient cells. Increasing evidence[11, 12] has shown that exosomal miRNAs are the key elements involved in the pathogenesis of breast cancer. Thus, a better understanding of the role of exosomal miRNAs in breast cancer pathogenesis could contribute to the development of new therapeutic methods. Furthermore, it 
has been proven that exosomal miRNAs can be used as noninvasive biomarkers in diagnosis and monitoring therapy in breast cancer[13-15].

In this study, we aimed to analyze the DE miRNAs in serum exosomes derived from patients with breast cancer and healthy control. We revealed different miRNA profiles of the exosomes from the breast cancer and healthy control groups. Additionally, using bioinformatic analysis we also predicted their potential pathogenic target genes, which might play roles in breast cancer development and progression. In particular, we studied the role of miR-1246 in invasion and migration by targeting THRB in MDA-MB-231 cells. Overall, our study provides important evidence and improved understanding of exosomal miRNAs in the process of breast cancer.

\section{Materials And Methods}

\section{Clinical characteristics of samples}

The patients recruited in this study underwent surgery at the Taizhou Central Hospital between 2018 and 2019. None of the patients had been treated by chemotherapy or radiotherapy prior to surgery and provided informed consent to participate in the study. Serum samples from 3 patients with breast cancer and control serum samples from 3 healthy female volunteers were obtained from the Taizhou Central Hospital.

\section{Profiling and validation of exosomal miRNAs in human serum samples}

To comprehensively profile exosomal miRNAs, exosomes were isolated from serum samples, and small RNA-seq libraries were prepared for high-throughput sequencing (Fig. 1A). The obtained exosomes were confirmed by transmission electron microscopy (TEM). Nanoparticle tracking analysis (NTA) was performed to characterize the exosomes. The raw sequencing data were first cleaned by removing both $5^{\prime}$ and 3 ' adaptors, and only high-quality reads were mapped to the human genome and annotated. The DbDEMC 2.0 database was used to find DE miRNAs in human cancers online (http://www.picb.ac.cn/dbDEMC/).

\section{Prediction and functional analysis of exosomal miRNA target genes}

MiRWalk 3.0 (http://mirwalk.umm.uni-heidelberg.de/), as a comprehensive archive, was used in our study to explore miRNA-target interaction data. To infer the potential functions of the DE miRNAs, Gene Ontology (GO) biological process and Kyoto Encyclopedia of Genes and Genomes (KEGG) analyses were performed. We performed functional analysis of these target genes using the STRING database (https://string-db.org/). Functional categories were clustered using the Functional Annotation Clustering tool, and representative GO categories and KEGG pathways are shown. 


\section{Cell culture and transfection}

MDA-MB-231 cells were maintained in DMEM (Sigma-Aldrich, St Louis, MO, USA). All cultures were grown in media supplemented with $10 \%$ fetal bovine serum (FBS, Sigma-Aldrich). The cells were cultured at $37^{\circ} \mathrm{C}$ with $5 \% \mathrm{CO}_{2}$.

MiR-1246 mimics and miR-224-5p NC were purchased from Synbio Tec (Suzhou, China). The breast carcinoma cells were prepared with Lipofectamine for small RNA transfections. The miRNA sequences were as follows:

miR-1246 mimics: 5'-AAUGGAUUUUUGGAGCAGG-3'

miR-1246 NC: 5'-ACGUGACACGUUCGGAGAAUU-3'.

\section{Cell invasion assay}

A 24-well transwell invasion chamber with Matrigel-coated filters was used for the cell invasion assay. Cells $\left(1 \times 10^{5} /\right.$ well) were plated into the top chamber, and culture medium containing $10 \%$ FBS was placed in the bottom chamber. The cells were fixed for $30 \mathrm{~min}$ in $4 \%$ paraformaldehyde and stained for 30 min with $0.1 \%$ hematoxylin after $24 \mathrm{~h}$. After observing the cell staining, the cell invasion was visualized with a microscope.

\section{Scratch assay}

A scratch assay was used to assess cell migration. MDA-MB-231 cells were transfected with $20 \mathrm{nM} \mathrm{miR-}$ 1246 or NC RNA in a 6-well plate. The cell monolayer at $90 \sim 95 \%$ confluence was scratched with a tip. Cells were then further cultured in a cell culture incubator. The average extent of wound closure was quantified at different time points.

\section{Colony formation assay}

The transfected cells were seeded into 6-well plates at a density of 2000 cells per well in DMEM containing FBS. The colony formation test was conducted at $10 \%$ for 10 days. Colonies were imaged and counted after fixation and staining.

\section{Kaplan-Meier plotter analysis}

Kaplan-Meier plotter is a database combining miRNA expression data and clinical information[16]. To evaluate the prognostic value of miR-1246 in breast cancer, Kaplan-Meier survival plots were generated. The hazard ratio (HR) with 95\% confidence intervals and log-rank P values were calculated.

\section{Statistical analysis}

Data were presented as mean \pm SD. All statistical analyses were performed using GraphPad Prism ((Version8, GraphPad Software, Inc. La Jolla, USA). The data sets were analyzed by Student's t test. A p value of less than 0.05 was considered statistically significant. 


\section{Results}

\section{Exosomal miRNA expression profiling of breast cancer serum}

RNA-seq was performed to generate the expression profiles of exosomal miRNAs in the serum samples from 3 breast cancer patients and 3 healthy controls. Exosomes isolated from the samples were confirmed by transmission electron microscopy (TEM) (Fig. 1B). Nanoparticle tracking analysis (NTA) was performed to characterize exosomes and show their size (Fig. 1C).

A total of $718 \mathrm{DE}$ miRNAs in exosomes were obtained between breast cancer and healthy controls (Fig. 1D). The top ten miRNAs were hsa-miR-146a-5p, hsa-miR-151a-3p, hsa-miR-146b-5p, hsa-miR-71103p, hsa-miR-221-3p, hsa-miR-432-5p, hsa-miR-451a, hsa-miR-3916, hsa-miR-1246, and hsa-miR-224-5p

(Table 1). MiRNAs were considered upregulated when they were increased by more than 2.5 -fold with a $p$ $<0.005$.

Table 1

Differentially expressed miRNAs between breast cancer and health control

\begin{tabular}{|llll|}
\hline Exosomes & \multicolumn{3}{c|}{ Serum and tissue } \\
\hline miRNA & Expression change & miRNA & Expression change \\
\hline hsa-miR-146a-5p & Down & hsa-miR-132-5p & Down \\
\hline hsa-miR-151a-3p & Down & hsa-miR-125b-1-3p & Down \\
hsa-miR-146b-5p & Down & hsa-miR-34c-5p & Down \\
hsa-miR-7110-3p & Up & hsa-miR-382-3p & Down \\
hsa-miR-221-3p & Down & hsa-miR-485-5p & Down \\
hsa-miR-432-5p & Down & hsa-miR-323b-3p & Down \\
\hline hsa-miR-451a & Up & hsa-miR-598-3p & Down \\
hsa-miR-3916 & Up & hsa-miR-224-5p & Up \\
hsa-miR-1246 & Up & hsa-miR-1246 & Up \\
hsa-miR-224-5p & Up & hsa-miR-184 & Up \\
\hline
\end{tabular}

Cellular functions and pathways can be affected by the complex interactions between exosomal miRNAs and their target genes. MiRNAs usually regulate more than one target gene. Hence, a many-to-many interaction relationship is normally observed between miRNAs and their target genes. Target genes of 10 miRNAs were predicted based on the miRWalk database, and 501 target genes were identified. Cytoscape was used to construct the miRNA-mRNA interaction network. Our results show a complex network consisting of the DE miRNAs and their target genes (Fig. 2A). 


\section{Functional analysis of the differentially expressed exosomal miRNAs}

Gene Ontology analysis showed that DE exosomal miRNAs are involved in various regulatory processes, such as the canonical Wnt signaling pathway, cell growth, autophagy, and neuron migration (Fig. 2B). KEGG analysis demonstrated that these exosomal miRNAs were involved in many cancers and pathways, such as breast cancer, Ras signaling pathway, Wnt signaling pathway, and autophagy (Fig. 2C). The above results show that these exosomal miRNAs play a crucial role in tumorigenesis by impacting various biological processes.

Compared with our previous study[17], 2 overlapping miRNAs were selected from data on serum exosomes, serum samples and tumor tissue: hsa-miR-1246 and hsa-miR-224-5p (Fig. 1E).

\section{MiR-1246 enhanced the migration and invasion of MDA-MB- 231 cells}

Transwell assays showed that MDA-MB-231 cell invasive ability was significantly promoted in the group transfected with miR-1246 mimics compared with the NC group $(p<0.05$, Fig. $3 A)$. Scratch test results indicated that miR-1246 mimics affected on MDA-MB-231 cell migration. As shown in Fig. 3B, a significantly delayed wound closure of MDA-MB-231 cells was observed in the NC group. The results of the clonogenic assay showed that miR-1246 did not affect the proliferation of MDA-MB-231 cells (Fig. 3C).

THRB is a target gene of miR-1246 (Fig. 4A). The level of THRB was determined after treatment of MDAMB-231 cells with miR-1246 mimics or miR-1246 NC. This finding shows that THRB expression decreased significantly after miR-1246 mimic treatment ( $p<0.05$, Fig. 4B).

\section{The prognostic value of miR-1246 in breast cancer}

The Kaplan-Meier plotter database was used to investigate the prognostic potential of miR-1246 expression in breast cancer. This analysis included 954 patients with breast cancer metastasized to the lymph nodes and 1024 patients with nonmetastatic breast cancer. From the results, miR-1246 was strongly correlated with lymph node status: positive with lymph node status [HR $=1.78(1.35-2.34), p=$ 3.6e-05] (Fig. 4C) and negative with lymph node status [HR=1.39 (1.01-1.91), $p=0.043$ ] (Fig. 4D). The detailed relationships between miR-1246 expression and prognosis of breast carcinoma based on different clinicopathological characteristics in the Kaplan-Meier plotter database are presented in Table 2. We found that miR-1246 was negatively correlated with overall survival (OS) in patients with breast cancer, especially in those with metastatic breast cancer. 
Table 2

Correlation of miR-1246 expression and clinical prognosis in breast cancer with different clinic pathological factors by Kaplan-Meier plotter

\begin{tabular}{|llll|}
\hline Clinicopathological characters & \multicolumn{3}{l}{ OS (Overall Survival) } \\
\cline { 2 - 4 } & N & HR & P \\
\hline Total & 1262 & $1.52(1.23-1.87)$ & $9 \mathrm{e}-05$ \\
\hline Molecular subtype & & & \\
\hline TNBC & 300 & $1.17(0.74-1.86)$ & 0.49 \\
\hline Luminal A & 546 & $1.57(1.09-2.26)$ & 0.014 \\
\hline Luminal B & 433 & $1.47(1.07-2.03)$ & 0.018 \\
\hline HER2 + ER- & 105 & $0.56(0.28-1.11)$ & 0.09 \\
\hline Grade & & & \\
\hline 1 & 103 & $2.69(1.11-6.53)$ & 0.023 \\
\hline 2 & 510 & $1.57(1.1-2.23)$ & 0.012 \\
\hline 3 & 774 & $1.45(1.11-1.91)$ & 0.0069 \\
\hline Systemically treated patients & & & \\
\hline ER-positive endocrine therapy & 779 & $1.54(1.15-2.07)$ & 0.0039 \\
\hline Chemotherapy & 280 & $1.66(1.09-2.53)$ & 0.0017 \\
\hline
\end{tabular}

\section{Discussion}

In the present study, differential miRNA expression profiles of serum exosomes were characterized in breast cancer patients and healthy controls. In total, $718 \mathrm{DE}$ exosomal miRNAs were identified. The top ten up-/downregulated miRNAs were selected. Five of them were unregulated (hsa-miR-7110-3p, hsa-miR451a, hsa-miR-3916, hsa-miR-1246, and hsa-miR-224-5p), and 5 of them were downregulated (hsa-miR146a-5p, hsa-miR-151a-3p, hsa-miR-146b-5p, hsa-miR-221-3p and hsa-miR-432-5p). We performed functional analyses of the ten DE exosomal miRNAs by the GO and KEGG databases. The results revealed that these miRNAs are involved in several regulatory processes, playing a crucial role in tumorigenesis by impacting various biological processes. For instance, hsa-miR-146a-5p functions as a tumor suppressor in various types of cancer[18, 19], hsa-miRNA-146a-5p could inhibit the proliferation and metastasis of breast cancer cells[20, 21], and hsa-miR-151a-3p expression was found to be decreased in many diseases[22]. The dysregulation of miRNAs, such as hsa-miR-146b-5p, hsa-miR-221$3 p$, hsa-miR-432-5p, hsa-miR-451a, hsa-miR-1246 and hsa-miR-224-5p, has been documented in a variety of human diseases and malignancies[23-31]. 
When the results were compared with our previous study[17], 2 overlapping DE miRNAs were selected: hsa-miR-1246 and hsa-miR-224-5p. In our previous study, hsa-miR-224-5p was shown to inhibit autophagy in breast cancer[31]. In this investigation, cell invasion assays, colony formation assays and scratch assays were performed to analyze the role of miR-1246 in breast carcinoma. The results revealed that miR-1246 enhanced the invasion and migration of breast cancer cells. However, miR-1246 did not affect the proliferation of MDA-MB-231 cells. To elucidate the molecular mechanisms of miR-1246mediated tumorigenesis, the level of THRB, one of the targets of miR-1246, was determined. The results showed that the expression of THRB was decreased after miR-1246 mimic treatment in MDA-MB-231 cells. Studies by Martines Iglesias and others have found that hypothyroidism can inhibit the growth of breast cancer cells and simultaneously enhance the invasion and metastasis of breast cancer cells[32, 33].

THRB, thyroid hormone receptor beta, is closely related to the occurrence and development of various human tumors, but its role and mechanism in breast cancer are not yet clear. The breast is a hormonedependent organ, and changes in the function of the endocrine system are closely related to the occurrence of breast diseases. Many endocrine hormones and their receptors also regulate it. In recent years, the relationship between thyroid hormones and their receptors and breast cancer has been widely studied[34-37]. This study found that in MDA-MB-231 cells, overexpression of miR-1246 inhibited the expression of THRB, thereby affecting migration and invasion. The specific mechanism is not yet clear. This may be due to the partial similarity between thyroid hormone receptors and estrogen receptors in the arrangement of nucleic acids. These molecules have a common pathway in regulating the growth of target cells, and the strength of the two effects may determine the direction of the development of breast disease[38]. To clarify the specific molecular mechanism, further research will be conducted in the future.

Furthermore, the Kaplan-Meier plotter was used to analyze the prognostic value of miR-1246 in breast cancer. The result suggested that the expression of miR-1246 was negatively correlated with OS in patients with breast cancer, especially in those with metastatic breast cancer. Hence, miR-1246 is an important molecule that could be a useful potential prognostic biomarker.

\section{Conclusion}

In conclusion, the current study identified DE exosomal miRNAs that may be involved in the pathogenesis of breast cancer based on RNA sequencing and bioinformatics data. This study provided a theoretical basis to further elucidate the biological function of miR-1246 in the progression of breast cancer.

\section{Abbreviations}

DE: Differentially expressed; ER: Estrogen receptor; Her2/ErBb2: Human epidermal growth factor receptor; miRNAs: microRNAs; TEM: Transmission electron microscopy; NTA: Nanoparticle tracking analysis; GO: Gene Ontology; KEGG: Kyoto Encyclopedia of Genes and Genomes; HR: Hazard ratio; OS: Overall survival 


\section{Declarations}

Acknowledgements

Not applicable.

\section{Authors' contributions}

Jie Zhu designed the study; Xiaoying Chen interpreted the data and prepare the manuscript for publication; Fengxiang Xi supervised the data collection; Lei Zhao analyzed the data; Yichao Wang supervised the data collection; Jiaogui Xie analyzed the data, and reviewed the draft of the manuscript. All authors have read and approved the manuscript.

\section{Funding}

Not applicable.

\section{Availability of data and materials}

All data generated or analyzed during this study are included in this published article.

\section{Ethics approval and consent to participate}

Ethical considerations involved in this study have been addressed according to the Helsinki Declaration 2013. All Samples were obtained after informed consent. Ethical approval was obtained from the Research Ethics Committee of the TaiZhou Central Hospital (Taizhou University Hospital).

\section{Consent for publication}

Written informed consent was obtained from a legally authorized representative(s) for anonymized patient information to be published in this article.

\section{Competing interests}

No potential conflicts of interest were disclosed.

\section{References}

1. Jerusalem G, Lancellotti P, Kim SB: HER2 + breast cancer treatment and cardiotoxicity: monitoring and management. Breast Cancer Res Treat 2019, 177:237-250.

2. Ahmed M, Rubio IT, Klaase JM, Douek M: Surgical treatment of nonpalpable primary invasive and in situ breast cancer. Nat Rev Clin Oncol 2015, 12:645-663. 
3. Takahashi TA, Lee Cl, Johnson KM: Breast cancer screening: Does tomosynthesis augment mammography? Cleve Clin J Med 2017, 84:522-527.

4. Parise CA, Caggiano V: Risk of mortality of node-negative, ER/PR/HER2 breast cancer subtypes in T1, T2, and T3 tumors. Breast Cancer Res Treat 2017, 165:743-750.

5. Wesseling J, Tinterri C, Sapino A, Zanconati F, Lutke-Holzik M, Nguyen B, Deck KB, Querzoli P, Perin T, Giardina $C$, et al: An international study comparing conventional versus mRNA level testing (TargetPrint) for ER, PR, and HER2 status of breast cancer. Virchows Arch 2016, 469:297-304.

6. Barile L, Vassalli G: Exosomes: Therapy delivery tools and biomarkers of diseases. Pharmacol Ther 2017, 174:63-78.

7. Pillay P, Moodley K, Moodley J, Mackraj I: Placenta-derived exosomes: potential biomarkers of preeclampsia. Int J Nanomedicine 2017, 12:8009-8023.

8. Zhang Y, Liu Y, Liu H, Tang WH: Exosomes: biogenesis, biologic function and clinical potential. Cell Biosci 2019, 9:19.

9. Jan AT, Rahman S, Khan S, Tasduq SA, Choi I: Biology, Pathophysiological Role, and Clinical Implications of Exosomes: A Critical Appraisal. Cells 2019, 8.

10. Ye FG, Song CG, Cao ZG, Xia C, Chen DN, Chen L, Li S, Qiao F, Ling H, Yao L, et al: Cytidine Deaminase Axis Modulated by miR-484 Differentially Regulates Cell Proliferation and Chemoresistance in Breast Cancer. Cancer Res 2015, 75:1504-1515.

11. Gonzalez-Villasana V, Rashed MH, Gonzalez-Cantú Y, Bayraktar R, Menchaca-Arredondo JL, VazquezGuillen JM, Rodriguez-Padilla C, Lopez-Berestein G, Resendez-Perez D: Presence of Circulating miR-145, miR-155, and miR-382 in Exosomes Isolated from Serum of Breast Cancer Patients and Healthy Donors. Dis Markers 2019, 2019:6852917.

12. Kia V, Mortazavi Y, Paryan M, Biglari A, Mohammadi-Yeganeh S: Exosomal miRNAs from highly metastatic cells can induce metastasis in non-metastatic cells. Life Sci 2019, 220:162-168.

13. Rodríguez-Martínez A, de Miguel-Pérez D, Ortega FG, García-Puche JL, Robles-Fernández I, Exposito J, Martorell-Marugan J, Carmona-Sáez P, Garrido-Navas MDC, Rolfo C, et al: Exosomal miRNA profile as complementary tool in the diagnostic and prediction of treatment response in localized breast cancer under neoadjuvant chemotherapy. Breast Cancer Res 2019, 21:21.

14. He Y, Deng F, Yang S, Wang D, Chen X, Zhong S, Zhao J, Tang J: Exosomal microRNA: a novel biomarker for breast cancer. Biomark Med 2018, 12:177-188.

15. Wang M, Ji S, Shao G, Zhang J, Zhao K, Wang Z, Wu A: Effect of exosome biomarkers for diagnosis and prognosis of breast cancer patients. Clin Transl Oncol 2018, 20:906-911. 
16. Lánczky A, Nagy Á, Bottai G, Munkácsy G, Szabó A, Santarpia L, Győrffy B: miRpower: a web-tool to validate survival-associated miRNAs utilizing expression data from 2178 breast cancer patients. Breast Cancer Res Treat 2016, 160:439-446.

17. Zhu J, Zheng Z, Wang J, Sun J, Wang P, Cheng X, Fu L, Zhang L, Wang Z, Li Z: Different miRNA expression profiles between human breast cancer tumors and serum. Front Genet 2014, 5:149.

18. Yuwen DL, Sheng BB, Liu J, Wenyu W, Shu YQ: MiR-146a-5p level in serum exosomes predicts therapeutic effect of cisplatin in non-small cell lung cancer. Eur Rev Med Pharmacol Sci 2017, 21:26502658.

19. Simanovich E, Brod V, Rahat MM, Rahat MA: Function of miR-146a-5p in Tumor Cells As a Regulatory Switch between Cell Death and Angiogenesis: Macrophage Therapy Revisited. Front Immunol 2017, 8:1931.

20. Si C, Yu Q, Yao Y: Effect of miR-146a-5p on proliferation and metastasis of triple-negative breast cancer via regulation of SOX5. Exp Ther Med 2018, 15:4515-4521.

21. Gao W, Hua J, Jia Z, Ding J, Han Z, Dong Y, Lin Q, Yao Y: Expression of miR-146a-5p in breast cancer and its role in proliferation of breast cancer cells. Oncol Lett 2018, 15:9884-9888.

22. Xiao H, Wei N, Su M, Xiong Z: Down-regulation of serum miR-151a-3p is associated with renal tissue activity in class IV lupus nephritis. Clin Exp Rheumatol 2019, 37:67-72.

23. Zhu $Y$, Wu G, Yan $W$, Zhan $H$, Sun $P$ : miR-146b-5p regulates cell growth, invasion, and metabolism by targeting PDHB in colorectal cancer. Am J Cancer Res 2017, 7:1136-1150.

24. Feng J, Wang M, Li M, Yang J, Jia J, Liu L, Zhou J, Zhang C, Wang X: Serum miR-221-3p as a new potential biomarker for depressed mood in perioperative patients. Brain Res 2019, 1720:146296.

25. Liu H, Xue L, Song C, Liu F, Jiang T, Yang X: Overexpression of circular RNA circ_001569 indicates poor prognosis in hepatocellular carcinoma and promotes cell growth and metastasis by sponging miR411-5p and miR-432-5p. Biochem Biophys Res Commun 2018, 503:2659-2665.

26. Liu Y, Lu C, Zhou Y, Zhang Z, Sun L: Circular RNA hsa_circ_0008039 promotes breast cancer cell proliferation and migration by regulating miR-432-5p/E2F3 axis. Biochem Biophys Res Commun 2018, 502:358-363.

27. Uchida A, Seki N, Mizuno K, Yamada Y, Misono S, Sanada H, Kikkawa N, Kumamoto T, Suetsugu T, Inoue H: Regulation of KIF2A by Antitumor miR-451a Inhibits Cancer Cell Aggressiveness Features in Lung Squamous Cell Carcinoma. Cancers (Basel) 2019, 11.

28. Xu R, Li H, Wu S, Qu J, Yuan H, Zhou Y, Lu Q: MicroRNA-1246 regulates the radio-sensitizing effect of curcumin in bladder cancer cells via activating P53. Int Urol Nephrol 2019, 51:1771-1779. 
29. Lin SS, Peng CY, Liao YW, Chou MY, Hsieh PL, Yu CC: miR-1246 Targets CCNG2 to Enhance Cancer Stemness and Chemoresistance in Oral Carcinomas. Cancers (Basel) 2018, 10.

30. Li J, Liu X, Li C, Wang W: miR-224-5p inhibits proliferation, migration, and invasion by targeting PIK3R3/AKT3 in uveal melanoma. J Cell Biochem 2019, 120:12412-12421.

31. Cheng Y, Li Z, Xie J, Wang P, Zhu J, Li Y, Wang Y: MiRNA-224-5p inhibits autophagy in breast cancer cells via targeting Smad4. Biochem Biophys Res Commun 2018, 506:793-798.

32. Martínez-Iglesias O, García-Silva S, Regadera J, Aranda A: Hypothyroidism enhances tumor invasiveness and metastasis development. PLoS One 2009, 4:e6428.

33. Carr FE, Tai PW, Barnum MS, Gillis NE, Evans KG, Taber TH, White JH, Tomczak JA, Jaworski DM, Zaidi SK, et al: Thyroid Hormone Receptor- $\beta$ (TR $\beta$ ) Mediates Runt-Related Transcription Factor 2 (Runx2) Expression in Thyroid Cancer Cells: A Novel Signaling Pathway in Thyroid Cancer. Endocrinology 2016, 157:3278-3292.

34. Vattai A, Akyol E, Kuhn C, Hofmann S, Heidegger H, von Koch F, Hermelink K, Wuerstlein R, Harbeck N, Mayr D, et al: Increased trace amine-associated receptor 1 (TAAR1) expression is associated with a positive survival rate in patients with breast cancer. $J$ Cancer Res Clin Oncol 2017, 143:1637-1647.

35. Park JW, Zhao L, Willingham MC, Cheng SY: Inhibition of STAT3 signaling blocks obesity-induced mammary hyperplasia in a mouse model. Am J Cancer Res 2017, 7:727-739.

36. Lin HY, Chin YT, Yang YC, Lai HY, Wang-Peng J, Liu LF, Tang HY, Davis PJ: Thyroid Hormone, Cancer, and Apoptosis. Compr Physiol 2016, 6:1221-1237.

37. Flamini MI, Uzair ID, Pennacchio GE, Neira FJ, Mondaca JM, Cuello-Carrión FD, Jahn GA, Simoncini T, Sanchez AM: Thyroid Hormone Controls Breast Cancer Cell Movement via Integrin aV/B3/SRC/FAK/PI3Kinases. Horm Cancer 2017, 8:16-27.

38. Topper YJ, Freeman CS: Multiple hormone interactions in the developmental biology of the mammary gland. Physiol Rev 1980, 60:1049-1106.

\section{Figures}


A

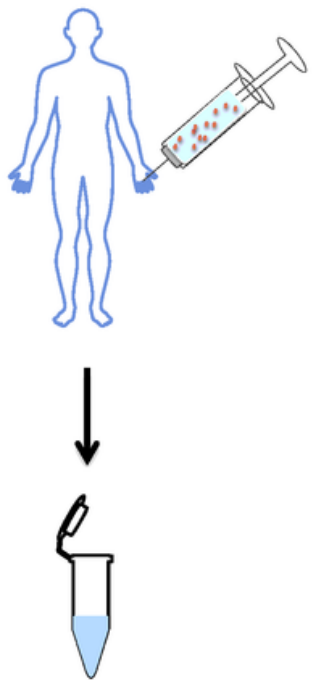

Exosome isolation and RNA extraction

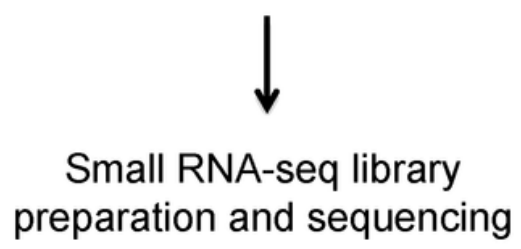

Small RNA-seq library preparation and sequencing
B

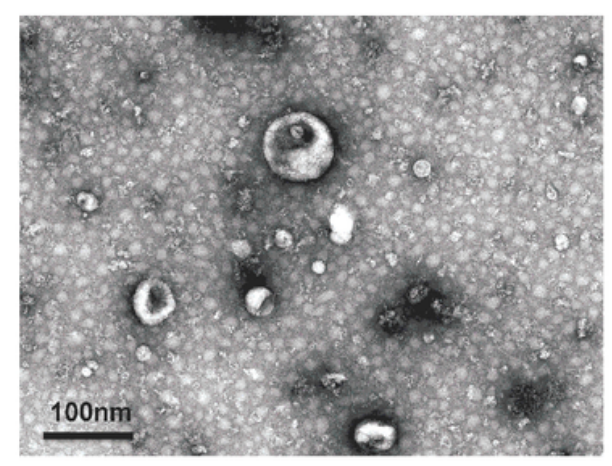

\section{D}

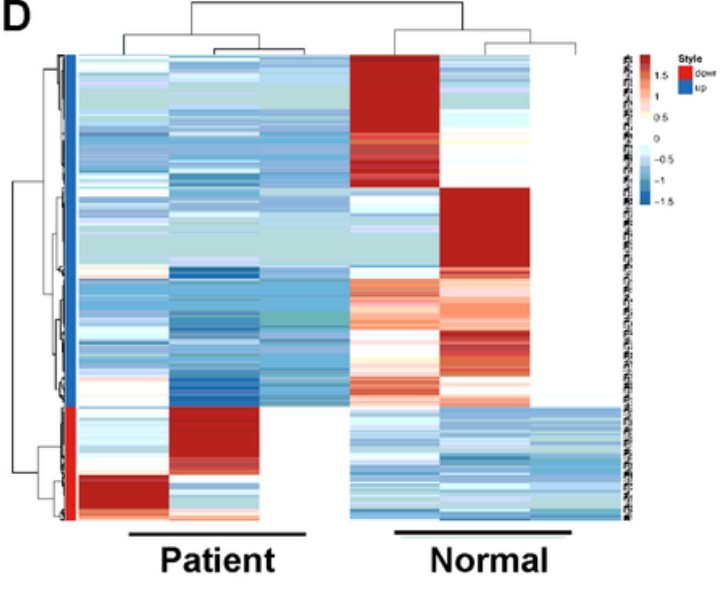

C

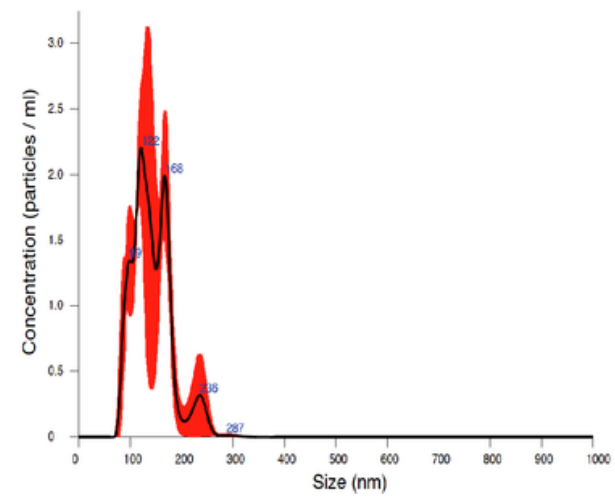

E Exosomes

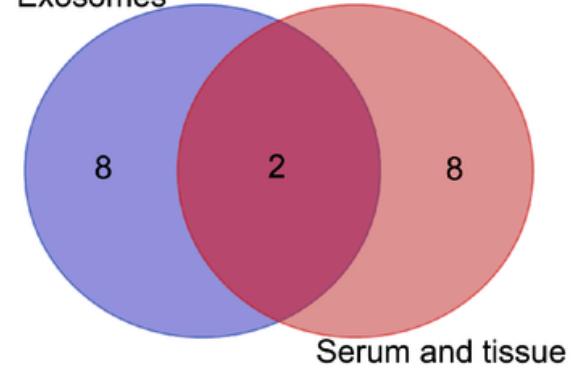

$\mathrm{miR}-1246$

miR-224-5p

\section{Figure 1}

Profiling of miRNAs in exosomes from the serum of breast cancer patients and healthy controls. (A) The procedure of exosome collection and RNA extraction. (B) Transmission electron micrograph (TEM) of exosomes isolated from serum (the scale bar is $100 \mathrm{~nm}$ ). (C) Nanoparticle tracking analysis (NTA) was used to determine vesicle concentration and size distribution. (D) A portion of the heat map of 718 differentially expressed miRNAs in exosomes between breast cancer and healthy controls is shown. Upregulated miRNAs are indicated in blue, and downregulated miRNAs are indicated in red. (E) Venn diagram showing two miRNAs (miR-1246 and miR-224-5p) that overlapped in the data from breast cancer serum exosomes, serum samples and tissues. 
A

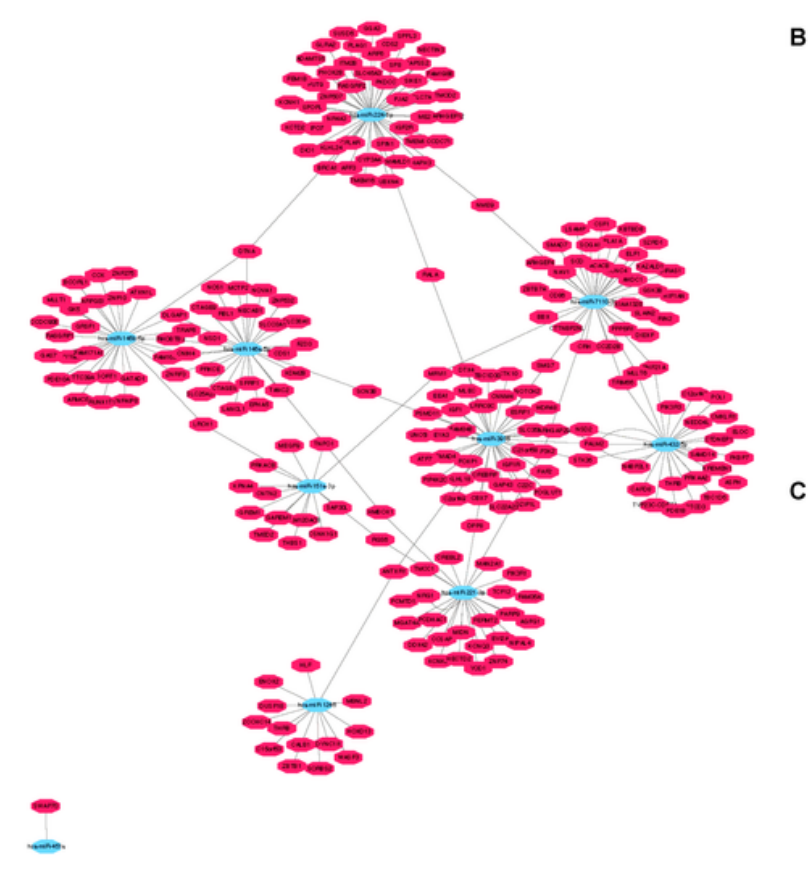

B
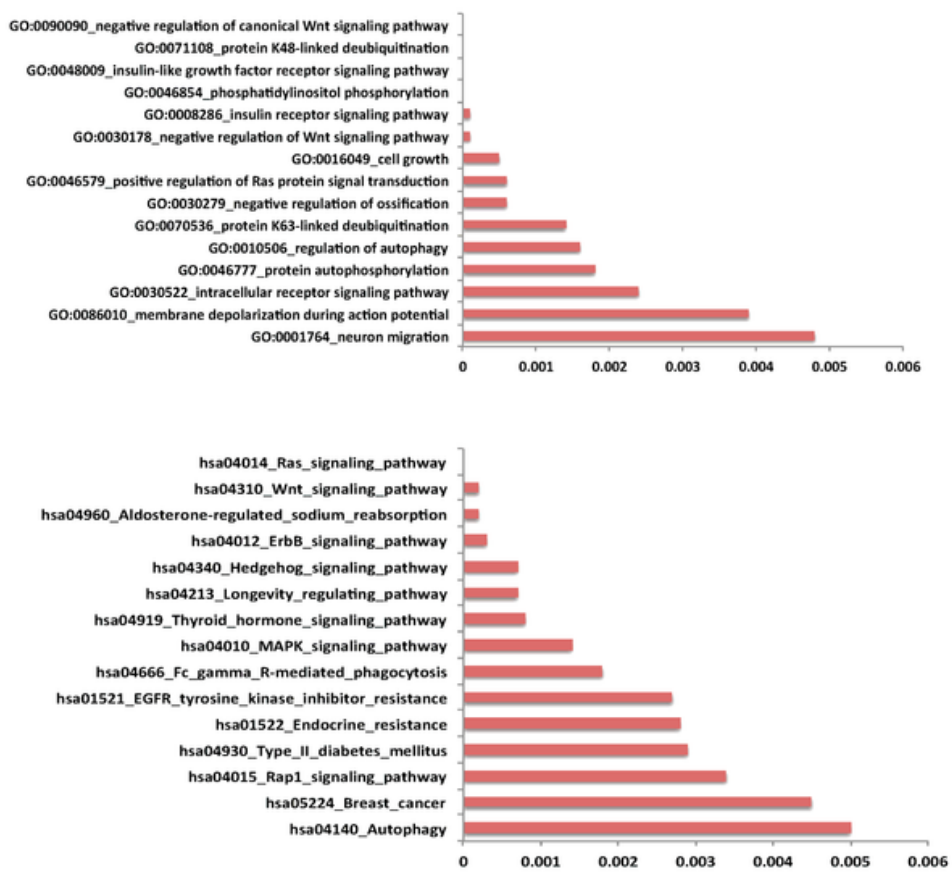

\section{Figure 2}

(A) The results of the network of the ten exosomal miRNAs and their target genes in breast cancer. The ten miRNAs are shown in blue, and target genes of the miRNAs are shown in red. (B) Top $15 \mathrm{GO}$ analysis terms of the top ten upregulated and downregulated exosomal miRNAs. (C) Top 15 KEGG pathways of the top ten upregulated and downregulated exosomal miRNAs. 
A

miRNA-NC

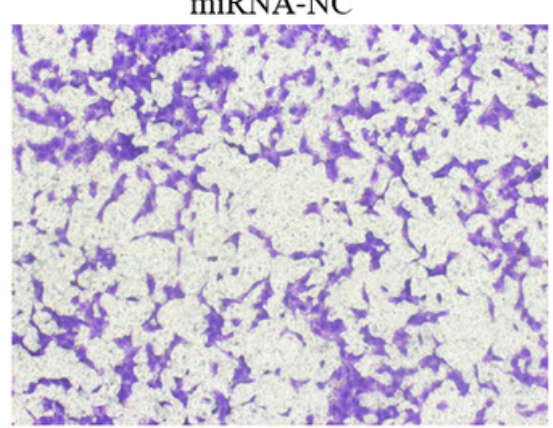

miRNA-1246

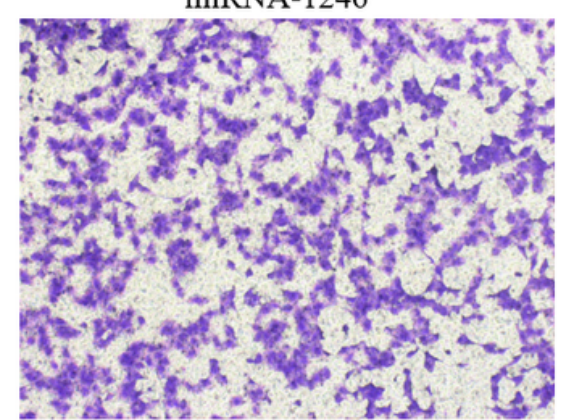

$48 \mathrm{~h}$

B
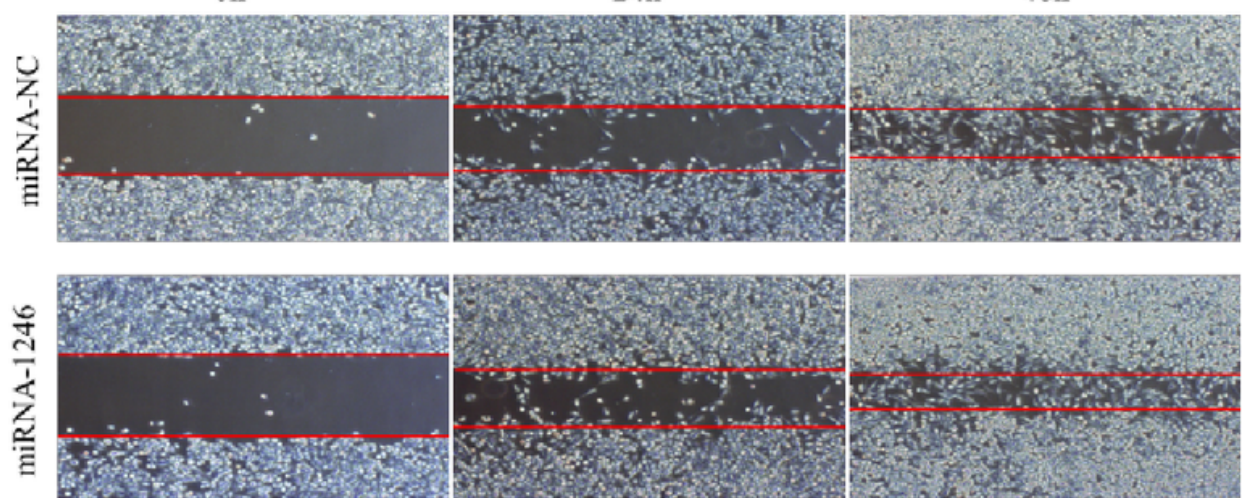

C
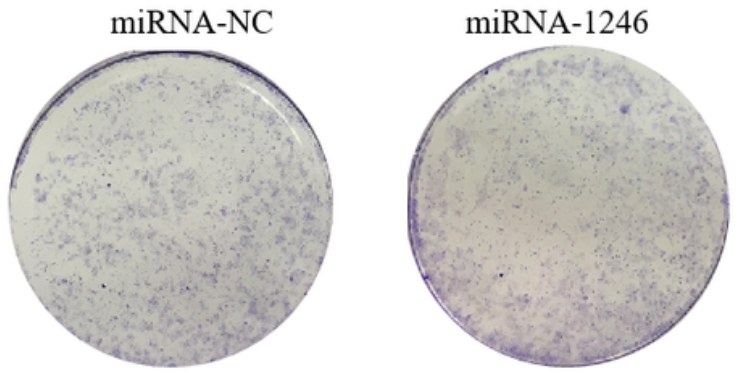
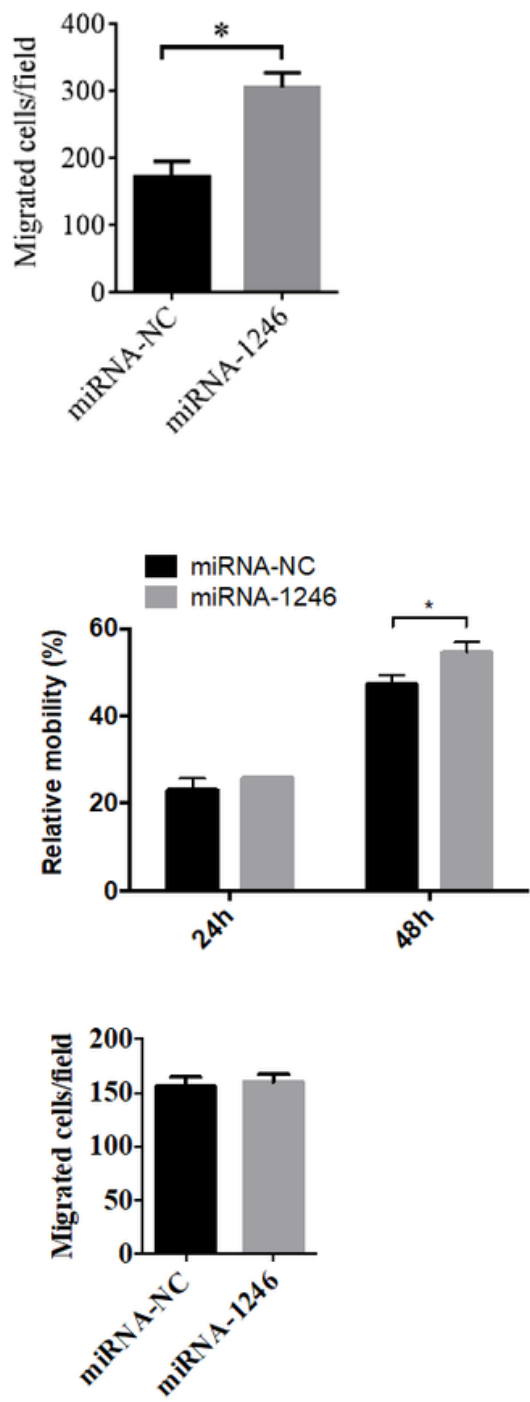

Figure 3

MiR-1246 enhanced the migration and invasion of breast cancer cells. (A) Transwell assays were used to analyze the invasiveness of breast cancer cells. (B) A scratch assay was used to verify the changes in migration of breast cancer cells. (C) A clonogenic assay was used to confirm the clonogenic variations of breast cancer cells. 
A

THRB 3'-UTR 5' ...AUAGUGAUAGACUAUAAUCCAUA...

has-miR-1246

3'

I| || | ||

GGACGAGGUUUUUAGGUAA

B

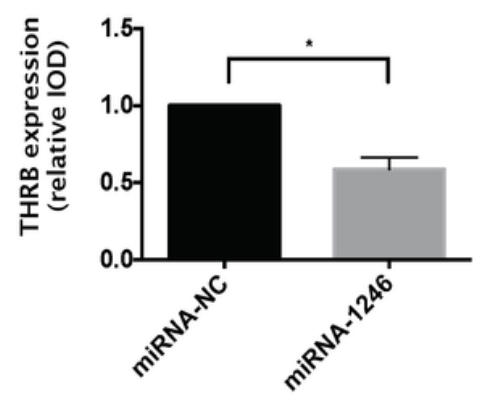

C

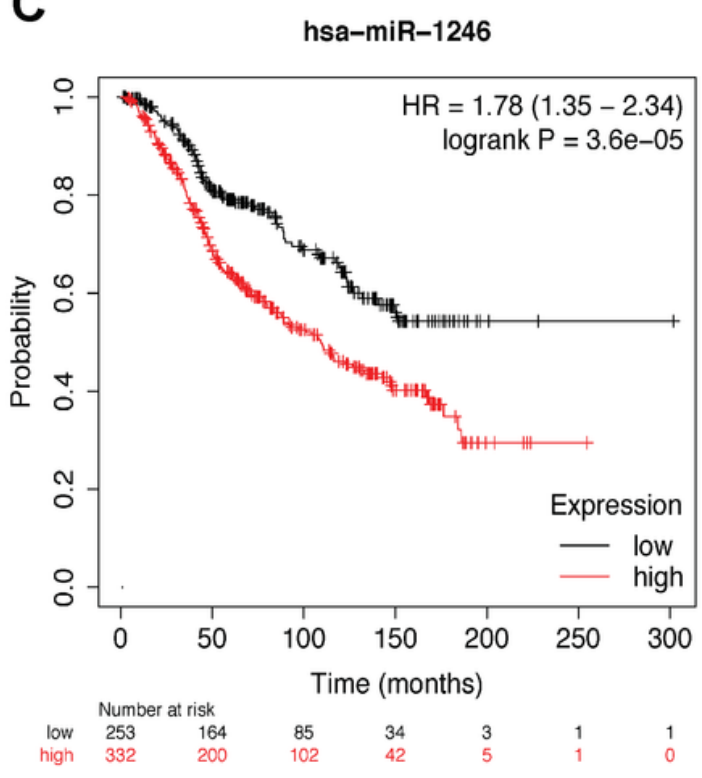

D

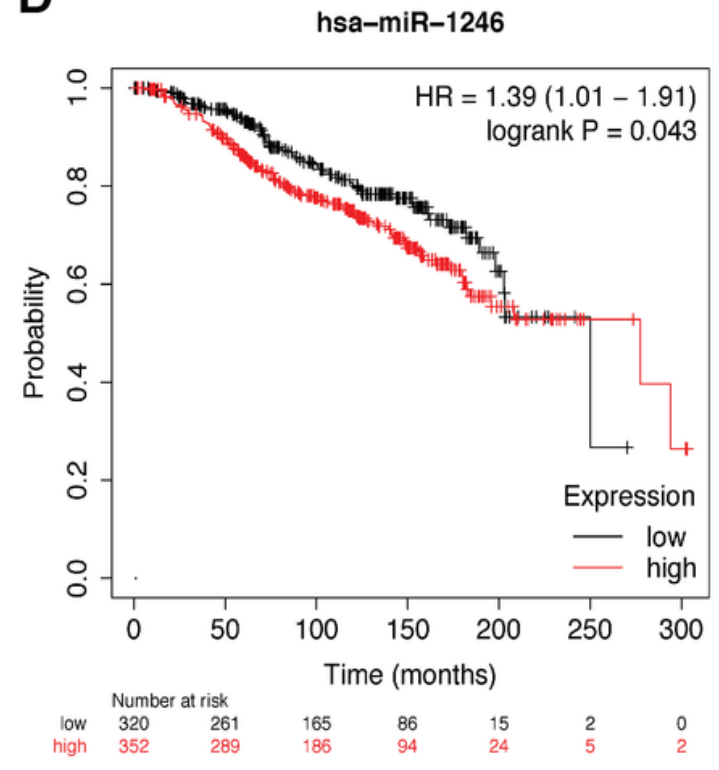

Figure 4

(A) THRB is a target gene of miR-1246. (B)The expression level of THRB in MDR-MB-231 cells which transfected with miR-224-5p mimics or miR-1246 NC, * $p<0.05$. Kaplan-Meier analysis was used to determine the prognostic role of miR-1246 in breast cancer. (C) Positive with lymph node status, (D) negative with lymph node status. 